\title{
The X-ray variability and the near-IR to X-ray spectral energy distribution of four low luminosity Seyfert 1 galaxies
}

\author{
I. E. Papadakis ${ }^{1,2}$, Z. Ioannou ${ }^{2,1}$, W. Brinkmann ${ }^{3,2}$, and E. M. Xilouris ${ }^{4}$ \\ 1 Physics Department, University of Crete, PO Box 2208, 71003 Heraklion, Crete, Greece \\ e-mail: jhep@physics.uoc.gr \\ 2 IESL, Foundation for Research and Technology, 71110 Heraklion, Crete, Greece \\ 3 Max-Planck-Institut für extraterrestrische Physik, Giessenbachstrasse, 85740 Garching, Germany \\ ${ }^{4}$ Institute of Astronomy and Astrophysics, National Observatory of Athens, I. Metaxa \& V. Pavlou, 15236 P. Penteli, Athens, Greece
}

Received 31 May 2008 / Accepted 26 August 2008

\section{ABSTRACT}

\begin{abstract}
Context. We present the results from a study of the X-ray variability and the near-IR to X-ray spectral energy distribution of four low-luminosity, Seyfert 1 galaxies.

Aims. We compared their variability amplitude and broad band spectrum with those of more luminous AGN in order to investigate whether accretion in low-luminosity AGN operates as in their luminous counterparts.

Methods. We used archival XMM-Newton and, in two cases, ASCA data to estimate their X-ray variability amplitude and determine their X-ray spectral shape and luminosity. We also used archival HST data to measure their optical nuclear luminosity, and near-IR measurements from the literature, in order to construct their near-IR to X-ray spectra.

Results. The X-ray variability amplitude of the four Seyferts is what one would expect, given their black hole masses. Their near-IR to X-ray spectrum has the same shape as the spectrum of quasars that are $10^{2}-10^{5}$ times more luminous.

Conclusions. The objects in our sample are optically classified as Seyfert 1-1.5. This implies that they host a relatively unobscured AGN-like nucleus. They are also of low luminosity and accrete at a low rate. They are therefore good candidates to detect radiation from an inefficient accretion process. However, our results suggest that they are similar to AGN that are $10^{2}-10^{5}$ times more luminous. The combination of a "radiative efficient accretion disc plus an X-ray producing hot corona" may persist at low accretion rates as well.
\end{abstract}

Key words. galaxies: active - galaxies: Seyfert - X-rays: galaxies

\section{Introduction}

The current paradigm for active galactic nuclei (AGN) comprises a supermassive black hole $(\mathrm{BH})$ which is accreting material through a disc. Accretion in a geometrically thin, optically thick disc (Shakura \& Sunyaev 1973) is the favored mechanism to achieve the high accretion efficiency (of the order of $\sim 0.1$ or so) that is necessary to explain the high power emitted by AGN. Theoretical modeling of low accretion rate systems suggests that, when the accretion rate is below a few percent of the Eddington limit, AGN may switch to a different accretion mode characterized by low radiative efficiency. Most "radiatively inefficient accretion flow" models (RIAFs; see, e.g. Yuan 2007, and references therein) suggest that the kinetic energy associated with the gas is either advected with the matter into the $\mathrm{BH}$ or redirected into an outflow.

Many of the nearby AGN are intrinsically faint (exhibiting bolometric luminosities less than $10^{43-44} \mathrm{erg} / \mathrm{s}$ or so) while the central $\mathrm{BH}$ in a large fraction of them is as large as $10^{7}-10^{8} \mathrm{M}_{\odot}$ (see, e.g. Panessa et al. 2006). Consequently, most of these nuclei accrete at a rate lower than a few percent of $L_{\text {Edd }}$. It is then possible that accretion in these objects operates in a radiative inefficient mode. For example, according to Merloni et al. (2003), the accretion mode should change below $L_{\mathrm{X}} / L_{\mathrm{Edd}} \sim 10^{-3}$ (where $L_{\mathrm{X}}$ and $L_{\mathrm{Edd}}$ are the $2-10 \mathrm{keV}$ and Eddington luminosity, respectively), while Chiaberge et al. (2005) suggested that RIAFs may operate in active nuclei with $L_{\mathrm{O}} / L_{\mathrm{Edd}} \leq 10^{-4}$ (where $L_{\mathrm{O}}$ is the optical luminosity in the $R$-band). However, conclusive evidence for the presence of RIAFs in low luminosity AGNs (LLAGNs) is still lacking.

One of the most frequently applied tests to investigate whether a RIAF operates in LLAGNs is to compare their nuclear spectral energy distribution (SED) in the near-infrared (IR) to ultraviolet (UV) part of the spectrum with the average SED of powerful quasars. At these frequencies different accretion disc models are expected to show large differences in spectral shape. RIAFs should lack both the "big blue bump" and the IR (reprocessed) bump, which instead characterize the emission from an optically thick, geometrically thin accretion disc and the surrounding heated dust. Application of this test to various samples of LLAGNs has yielded contradictory results. While Maoz (2007) found that the broad band SEDs of AGNs with luminosities as low as $\sim 10^{40} \mathrm{erg} / \mathrm{s}$ are quite similar to the SED of more luminous AGN, Ho (1999) found that the low-luminosity AGN SEDs have a weak or absent blue bump and are "radio loud". Quataert et al. (1999) found that the optical/UV spectrum of M81 and NGC 4579 decreases with increasing frequency, in contrast to the "canonical" quasar spectrum. Ptak et al. (2004) found that NGC 3998 (a "type 1" LINER galaxy) lacks the "big blue bump", while RIAF models can fit its UV to X-ray SED reasonably well. Chiaberge et al. (2006) also found that the SED of a low luminosity Seyfert 2 galaxy, namely NGC 4565, is different from that of luminous quasars.

Another powerful test to identify the presence of RIAFs in low luminosity AGNs is to compare their variability properties with the properties of more luminous AGNs. Ptak et al. (1998) 
Table 1. The XMM-Newton observations details.

\begin{tabular}{|c|c|c|c|c|c|c|c|}
\hline Object & $\begin{array}{c}\text { Observing date } \\
\text { UT }\end{array}$ & Instrument & ObsId & Mode & Filter & $\begin{array}{c}\text { Exposure } \\
(\mathrm{ks})\end{array}$ & $\begin{array}{c}\text { Count rate } \\
\text { cts } / \mathrm{s}^{(\ddagger)}\end{array}$ \\
\hline \multirow[t]{3}{*}{ NGC 4235} & Jun. 09, 2004 & $\overline{\mathrm{PN}}$ & \multirow[t]{3}{*}{0204650201} & Large Window & Thin & 9.6 & 0.96 \\
\hline & 09:04-12:43 & MOS1 & & Prime Partial & Thin & 10.9 & 0.31 \\
\hline & & MOS2 & & Prime Partial & Thin & 10.9 & 0.31 \\
\hline \multirow[t]{3}{*}{ NGC 4639} & Dec. 12, 2001 & $\mathrm{PN}$ & \multirow[t]{3}{*}{0112551001} & Extd. Full Frame & Medium & 8.8 & 0.28 \\
\hline & $16: 19-18: 19$ & MOS1 & & Full Window & Thin & 14.2 & 0.09 \\
\hline & & MOS2 & & Full Window & Thin & 14.2 & 0.09 \\
\hline \multirow[t]{3}{*}{ NGC 5033} & Dec. 18, 2002 & $\mathrm{PN}$ & \multirow[t]{3}{*}{0094360501} & Full Frame & Medium & 9.1 & 2.40 \\
\hline & 15:05-19:01 & MOS1 & & Full Window & Medium & 11.5 & 0.68 \\
\hline & & MOS2 & & Full Window & Medium & 11.5 & 0.68 \\
\hline \multirow[t]{3}{*}{ NGC 5273} & Jun. 14, 2002 & $\mathrm{PN}$ & \multirow[t]{3}{*}{0112551701} & Extd. Full Frame & Medium & 9.3 & 1.57 \\
\hline & $13: 15-16: 37$ & MOS1 & & Full Window & Thin & 14.2 & 0.57 \\
\hline & & MOS2 & & Full Window & Thin & 14.2 & 0.56 \\
\hline
\end{tabular}

\# Background subtracted values.

found that LLAGNs tend to show little or no significant shortterm variability, despite their low luminosity. This is opposite to what is observed in powerful AGN, where the variability amplitude increases with decreasing luminosity. This difference has been interpreted as an indication that the size of the X-ray producing region in LLAGNs is significantly larger than the size of the X-ray source in "normal" Seyferts. This is in support of the hypothesis that advection-dominated accretion operates in LLAGNs, as in this case one can explain the larger size of the nuclear source.

Our aim is to compare the X-ray variability amplitude and the optical/X-rays SED of low luminosity AGN and powerful quasars. We used the Palomar optical spectroscopic survey of nearby galaxies (Ho et al. 1995) to find suitable objects for our study. This sample has the advantage of having uniform and high-quality data that allowed classification of the galactic nuclei to be determined with well defined and objective criteria (Ho et al. 1997). We only considered objects which are classified as Seyfert 1-Seyfert 1.5, because in this case we can be certain of the AGN-like nature of their nuclear activity, and that the central source is unobscured. There are 11 such objects in the Palomar sample (Ho et al. 1997). We chose four from these sources, namely NGC 4235, NGC 4639, NGC 5033 and NGC 5273, because there exist estimates of their central black hole mass (hence we can estimate their $L_{\mathrm{Edd}}$ ) and their nuclear luminosity is low: their $L_{\mathrm{X}} / L_{\mathrm{Edd}}$ ratio is smaller than $10^{-3}$. Therefore, it is possible that accretion in these objects operates in a radiatively inefficient mode.

First, we used archival XMM-Newton and ASCA data to measure their X-ray variability amplitude and we compared it with the variability amplitude of more luminous Seyferts. Then, by i) fitting models to the XMM-Newton spectra of the four sources; ii) measuring their nuclear optical luminosity using archival $H S T$ data; and iii) using near-IR luminosity measurements from the literature, we constructed their near-IR to X-ray SEDs and we compared them with the mean, broad band SED of luminous PG quasars. Our final results that the properties of the four objects in our sample are similar to those of more luminous AGNs, should have interesting implications regarding the nature of the accretion mode in low luminosity active galaxies.

\section{The X-ray timing and spectral analysis}

\subsection{The XMM-Newton observations}

All four Seyferts were observed by XMM-Newton. Although the exposures are less than $\sim 15 \mathrm{ks}$ long, XMM-Newton can provide high quality data for the detection of short term variations and the accurate determination of their nuclear X-ray spectral shape and luminosity.

We have retrieved data from the public XMM-Newton archive. Details of the observations are given in Table 1. The EPIC data were reprocessed with the XMMSAS version 6.5. None of the observations were affected by strong background flaring events. With an average count rate of a few cts $\mathrm{s}^{-1}$ photon pile-up is negligible for the PN and the MOS detectors, as verified using the XMMSAS task epatplot.

For the PN instrument, source counts were accumulated from a rectangular box of $27 \times 26$ RAW pixels ( 1 RAW pixel $\sim 4.1^{\prime \prime}$ ) around the position of the source. Background data were extracted from a similar, source free region on the chip. We selected only single and double events (PATTERN $\leq 4$, FLAG $\leq 0$; for details of the instruments see Ehle et al. 2007) in the energy range from $400 \mathrm{eV}$ to $10 \mathrm{keV}$. For the MOS data we accumulated the source counts using a circular aperture with radius $60^{\prime \prime}$ centered on the source positions. The background data were extracted from a similar region on the same chip. Events with PATTERN $\leq 12$ and FLAG $=0$ were used for the analysis.

The XMM-Newton images for all objects show a bright, compact, unresolved nucleus. NGC 4639, NGC 5033 and NGC 5273 have also been observed with Chandra. The higher resolution Chandra images reveal the presence of a bright nucleus in all cases as well. Weak diffuse emission, which is less prominent in the hard band, appears in the images of NGC 4639 and NGC 5033. There are two off-nuclear sources (at a distance of less than $\sim 1$ arcmin) in the case of NGC 5033 and one such source in NGC 5273. These sources are significantly weaker than the central nucleus in both cases. We therefore expect that the XMM-Newton data should not be significantly affected by contamination from either unresolved sources or diffuse emission.

\subsection{Timing analysis}

We created $0.4-10 \mathrm{keV}$ band light curves by adding the MOS1 and MOS2 data only. Although the PN light curves have a better signal to noise ratio, they are shorter than the MOS light curves. Since the amplitude of the X-ray variations in AGN generally increases with increasing time scale, we decided to use the longest possible light curves ${ }^{1}$. We binned the combined MOS light curves into intervals of size $500 \mathrm{~s}$. In this way the

\footnotetext{
1 The PN and MOS light curves look similar in the time intervals where both instruments were in operation.
} 


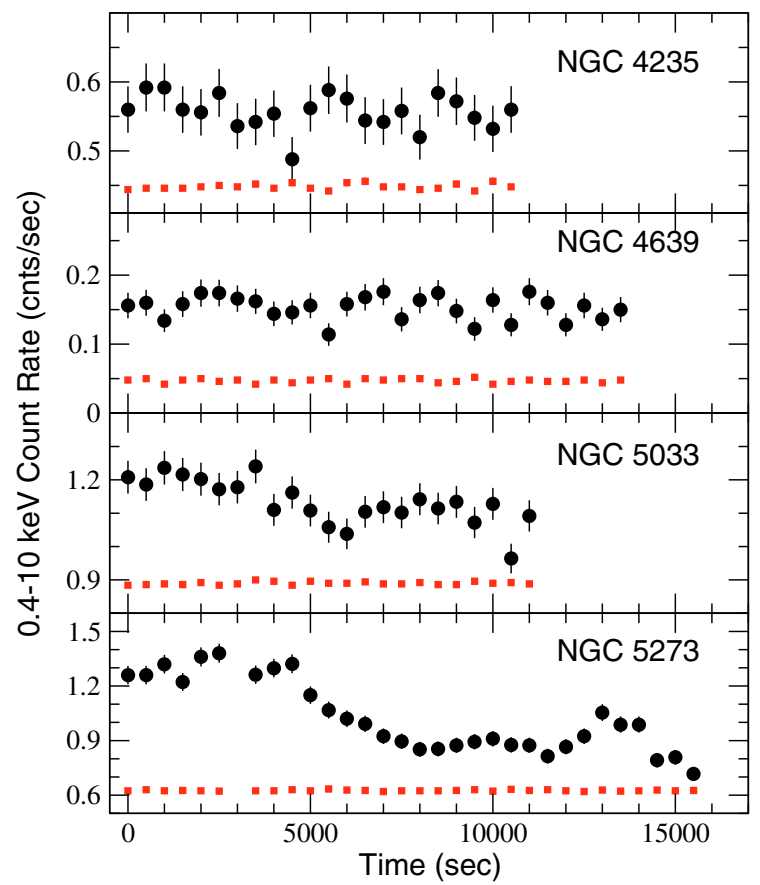

Fig. 1. Background subtracted, combined MOS1+2, 0.4-10 keV light curves of the four sources, binned in $500 \mathrm{~s}$ intervals. Time is measured from the start of each observation. Filled squares in all panels indicate the respective background light curves, rescaled appropriately in each case (errors are smaller than the symbol size).

number of photons in each bin is large enough (even in the case of the faintest source, NGC 4639) to guarantee the applicability of the traditional $\chi^{2}$ test to examine whether a source is variable or not. We accept that a light curve shows significant variations if the probability of the "null hypothesis" (i.e. the source is not variable) is less than $5 \%$.

The 0.4-10 keV, MOS1+2 light curves are shown in Fig. 1. NGC 4235 and NGC 4639 do not show significant variations: $\chi_{4235}^{2}=15.07 / 21$ degrees of freedom (d.o.f.), and $\chi_{4639}^{2}=$ $26.1 / 27$ d.o.f., respectively. On the other hand we do observe significant variations in the NGC 5503 and NGC 5273 light curves $\left(\chi_{5033}^{2}=44.3 / 22\right.$ and $\chi_{5273}^{2}=552 / 30$ d.o.f., respectively $)$.

We use the normalized excess variance, $\sigma_{\text {NXS }}^{2}$ (e.g. Nandra et al. 1997) as a measure of the intrinsic variability amplitude of the light curves which show significant variations. This estimator is defined as

$\sigma_{\mathrm{NXS}}^{2}=\frac{S^{2}-\left\langle\sigma_{\mathrm{err}}^{2}\right\rangle}{\langle x\rangle^{2}}$,

where $\langle x\rangle$, and $S^{2}=\left(1 / N_{\text {data }}\right) \sum_{i=1}^{N_{\text {data }}}\left(x_{i}-\langle x\rangle\right)^{2}$ are the mean and variance of the light curve, while $\left\langle\sigma_{\text {err }}^{2}\right\rangle=\left(1 / N_{\text {data }}\right) \sum_{i=1}^{N_{\text {data }}} \sigma_{\text {err }, i}^{2}$ is the average contribution of the Poisson noise process to the observed scatter around the mean $\left(N_{\text {data }}\right.$ is the number of points in the light curve). Its square root (the so called "fractional root mean square amplitude", $f_{\text {rms }}$ ) indicates the average variability amplitude of a source as a fraction of the light curve mean. We find that $f_{\text {rms }, 5033}=4 \pm 1 \%$ and $f_{\text {rms }, 5273}=18 \pm 1 \%$ (errors account only for the measurement errors in the light curve points and have been estimated according to the prescription of Vaughan et al. 2003).

In order to further investigate the variability of NGC 4235 and NGC 4639, we considered ASCA data as well. The main advantage of the ASCA light curves is that they are are longer

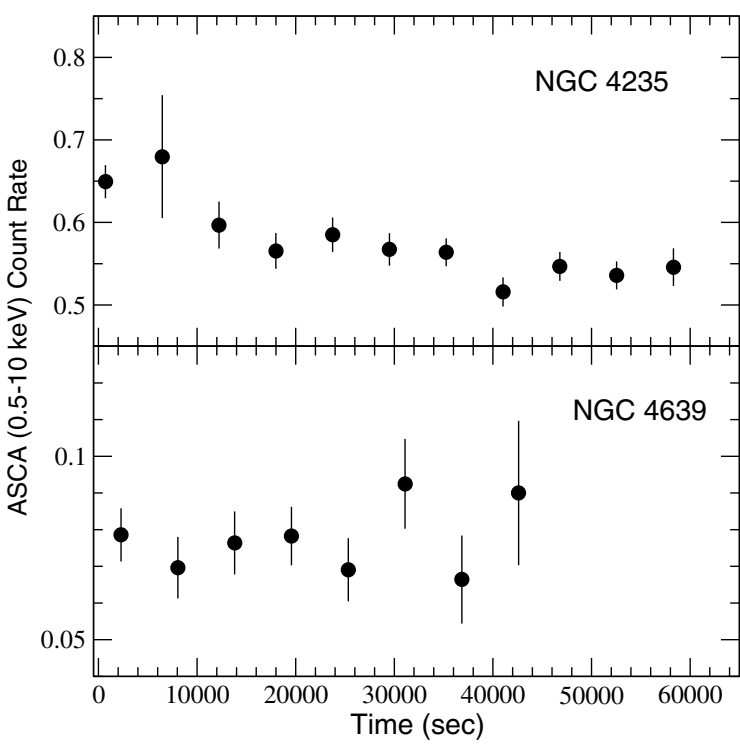

Fig. 2. Background subtracted, combined SIS $0+1,0.5-10 \mathrm{keV}$ band light curves of NGC 4235 and NGC 4639, binned in 5760 s intervals. Time is measured from the start of each observation.

than the XMM-Newton light curves. In the case of NGC 4235 , we employed its $0.5-10 \mathrm{keV}, 5760 \mathrm{~s}$ binned, SIS $0+1$ combined light curves from the $58 \mathrm{ks}$ long, December 1998 observation (sequence number 76005000; the light curves were downloaded from the TARTARUS database). The background subtracted light curve is plotted in the upper panel of Fig. 2. We detect significant variations $\left(\chi^{2}=39.8 / 10\right.$ d.o.f.) with $f_{\mathrm{rms}}, 0.5-10 \mathrm{keV}=6.2 \pm$ $1.8 \%$.

NGC 4639 has also been observed by ASCA. However, its background subtracted, $0.5-10 \mathrm{keV}$, SIS $0+1$ combined TARTARUS light curve (shown in the bottom panel of Fig. 2) does not reveal any significant variations during its $\sim 40 \mathrm{ks}$ observation in December 1998 (sequence number 76007000).

The top panel in Fig. 3 shows the logarithm of $\sigma_{\text {NXS }}^{2}$ plotted as a function of the logarithm of the X-ray (i.e., $2-10 \mathrm{keV}$ ) luminosity for nearby, bright Seyfert 1 nuclei (open circles; data are taken from O'Neill et al. 2005). The solid line is the best linear fit to the data. Filled squares in the same figure indicate the $\left[\log \left(\sigma_{\text {NXS }}^{2}\right), \log \left(L_{X}\right)\right]$ data of NGC 5273, NGC 5033 and NGC 4235 (using the X-ray luminosity estimates listed in Table 3). The thick arrow in this figure indicates the upper $3 \sigma$ upper limit of NGC 4639, as estimated using the MOS1+2 light curves. The plot suggests that, apart from NGC 5273, the other three Seyferts are less variable than what we would expect, if we consider the best line fit to the O'Neill et al. data.

However, there has been growing evidence that the excess variance in AGN is primarily related to $\mathrm{BH}$ mass (Papadakis 2004; O'Neill et al. 2005). If that is the case, then a "variability amplitude vs. luminosity" relation is expected only if the objects in the sample are accreting at a similar rate (Papadakis 2004). In the bottom panel of Fig. 3, we plot the logarithm of $\sigma_{\text {NXS }}^{2}$ as a function of the central black hole mass, $M_{\mathrm{BH}}$ (in Solar mass units), for the O'Neill et al. objects and the four Seyferts (BH mass estimates are listed in Table 2). This plot shows clearly that their variability amplitudes are in agreement with the amplitudes we measure in other Seyferts.

The fact that NGC 5273 shows the largest variability amplitude among the four objects in our sample is in agreement with its small black hole mass. It is not surprising that the NGC 4235 MOS light curve does not exhibit significant 

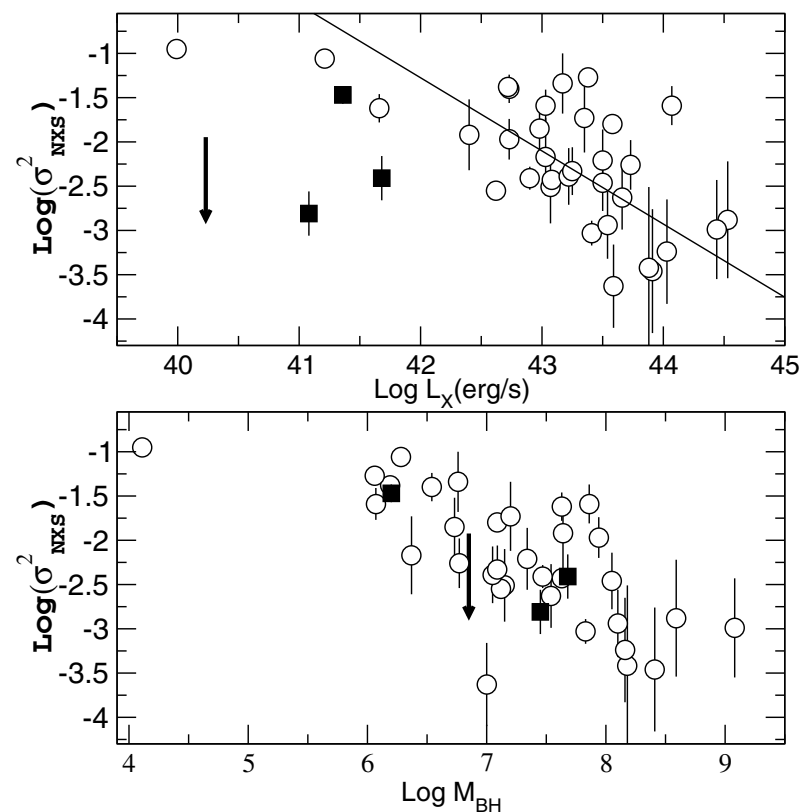

Fig. 3. Top panel: Log of $\sigma_{\text {NXs }}^{2}$ plotted as a function of the log of $\mathrm{X}$-ray luminosity for $33 \mathrm{AGN}$ (open circles; data are taken from O'Neill et al. 2005). Filled squares indicate the $\left[\log \left(\sigma_{\text {NXS }}^{2}\right), \log \left(L_{X}\right)\right]$ data of NGC 5273, NGC 5033 and NGC 4235, while the thick arrow indicates the $3 \sigma$ upper limit on $\log \left(\sigma_{\text {NXS }}^{2}\right)$ for NGC 4639. Bottom panel: the log of $\sigma_{\mathrm{NXS}}^{2}$ plotted as a function of the $\log (\mathrm{BH}$ mass) (in solar mass units) relation for the $33 \mathrm{AGN}$ of O'Neill et al. and the four objects in our sample.

variations, because it is the shortest of the light curves we study, and this object has the largest $M_{\mathrm{BH}}$ as well. The variations we observe in the $\sim 5.5$ times longer ASCA light curve are of the "correct" amplitude. As for NGC 4639, it is rather surprising (given its small black hole mass) that neither the MOS nor the $A S C A$ light curves reveal any significant variations. However, this is the faintest among the four sources, so the uncertainty in the measurement of its $\sigma_{\mathrm{NXS}}^{2}$ is quite large. We need a longer $X M M-N e w t o n$ observation to detect long term variations in this object.

Note that, in order to achieve the highest possible signalto-noise ratio, we have used full band light curves (i.e. either $0.4-10$ or $0.5-10 \mathrm{keV}$ ) to estimate $\sigma_{\text {NXS }}^{2}$, while O'Neill et al. use $2-10 \mathrm{keV}$ band light curves. However, this should not affect our results significantly, as $\sigma_{\mathrm{NXS}, 2-10 \mathrm{keV}}^{2}$ and $\sigma_{\mathrm{NXS}, 0.5-2 \mathrm{keV}}^{2}$ are comparable in AGN (Nandra et al. 1997). On the other hand, the NGC 5053 and NGC 5273 light curves are half the length of the light curves that O'Neill et al. use. We therefore conclude that the X-ray variability mechanism in these Seyferts operates in the same way as in more luminous AGN.

\subsection{X-ray spectral analysis}

For the X-ray spectral analysis, source spectra were grouped with a minimum of 20 counts per energy bin. Spectral fits have been performed with the XSPEC v11.3 package. Spectral responses and the effective area for the PN and MOS spectra were generated with the SAS commands rmfgen and arfgen. Individual fits to the PN and the MOS data resulted in similar model parameter values. For this reason, we present the results from the joint model fits to the PN and the MOS data in the $0.4-10 \mathrm{keV}$ energy band. In all model fits we add Galactic
Table 2. Distance and BH mass estimates.

\begin{tabular}{lccc}
\hline \hline Object & $\begin{array}{c}\text { Distance }^{a} \\
(\mathrm{Mpc})\end{array}$ & $\begin{array}{c}M_{\mathrm{BH}} \\
\left(M_{\odot}\right)\end{array}$ & Ref. $^{b}$ \\
\hline NGC 4235 & 38.0 & $4.8 \times 10^{7}$ & $(1)$ \\
NGC 4639 & 18.4 & $7.1 \times 10^{6}$ & $(2)$ \\
NGC 5033 & 15.2 & $2.8 \times 10^{7}$ & $(3)$ \\
NGC 5273 & 17.5 & $1.6 \times 10^{6}$ & $(4)$ \\
\hline
\end{tabular}

${ }^{a}$ Luminosity distances from NED; ${ }^{b}$ references for the black hole masses: (1) Dong \& de Robertis (2006); (2) Panessa et al. (2006); (3) Chiaberge et al. (2005); (4) Wu \& Han (2001).

interstellar absorption, using the $N_{\mathrm{H}}$ values reported by Dickey \& Lockman (1990). The best fitting model parameter values are presented together with their $90 \%$ errors in Table 3. In the same table we also list our derived $2-10 \mathrm{keV}$ fluxes and luminosities. As $K$-corrections can be neglected for these nearby objects we used the luminosity distances as given in NED (and listed in Table 2) for the calculation of the luminosities.

\subsubsection{NGC 4639 and NGC 5033}

Cappi et al. (2006) have already studied the XMM-Newton observations of these two sources, and our results are in agreement with theirs. In the case of NGC 4639, a simple power-law (PL) model with $\Gamma \sim 1.9$ plus cold absorption (with $N_{\mathrm{H}}$ fixed at the Galactic value) resulted in an acceptable fit to the full band spectra of all three instruments $\left(\chi_{\text {red }}^{2} /\right.$ d.o.f. $=1.11 / 230$; best fitting model values are listed in Table 3 ). We do not find an indication of a cold absorbing column in excess of the Galactic value, and the addition of a Gaussian line at $\sim 6.4 \mathrm{keV}$ does not significantly improve the goodness of the fit.

The full band spectrum of NGC 5033 can be fitted well by a power law plus a narrow Gaussian iron line, assuming galactic absorption only (the line's width in this case, as well as in the other sources below, is kept fixed at $0.01 \mathrm{keV}$, appropriate in the case of a narrow line). The addition of the line improves the fit quality by $\Delta \chi^{2}=44.1$ for 2 additional dof. The best model fitting parameters are listed in Table 3.

\subsubsection{NGC 5273}

The XMM-Newton observation of NGC 5273 has also been studied by Cappi et al. (2006). For this object our results do not agree with theirs, mainly because they consider the spectrum over a smaller energy band. A "PL plus Gaussian line plus Galactic absorption" model fits all the EPIC spectra well in the $2-10 \mathrm{keV}$ band. When extrapolated to lower energies, a strong, wide absorption feature appears around $\sim 1 \mathrm{keV}$. A number of complex models able to change the emission characteristics (like absorption edges, partial covering) resulted in fits of moderate quality $\left(\chi_{\text {red }}^{2} \leq 1.1\right)$, which show systematic deviations from the data in certain energy ranges.

Cappi et al. (2006) found that a PL plus a thermal plasma model with $\Gamma \sim 1.4$ and $k T \sim 0.2 \mathrm{keV}$ can fit the spectra well. We find that their model provides a good fit only in the "restricted" energy range of $0.6-10 \mathrm{keV}$, while at lower energies it diverges dramatically from the data. Excellent fits to all spectra over the $0.4-10 \mathrm{keV}$ energy range could be achieved by a PL plus a Gaussian line plus a thermal plasma emission model (parametrized with the component MEKAL in XSPEC), modified by the presence of an ionized absorber and cold Galactic 
Table 3. The X-ray model fit results.

\begin{tabular}{lcccc}
\hline \hline & $\mathrm{N} 4235$ & $\mathrm{~N} 4639$ & $\mathrm{~N} 5033$ & $\mathrm{~N} 5273$ \\
\hline$N_{\mathrm{H}, \mathrm{Gal}}^{\dagger}$ & 1.53 & 2.35 & 1 & 0.96 \\
$N_{\mathrm{H}, \text { int }}$ & $16 \pm 1$ & - & - & $94 \pm 4$ \\
$\mathrm{PL}$ & & & & \\
$\Gamma$ & 1.61 & 1.86 & 1.70 & 1.44 \\
& $( \pm 0.03)$ & $( \pm 0.04)$ & $( \pm 0.01)$ & $( \pm 0.03)$ \\
$A^{*}$ & 5.9 & 1.33 & 10.3 & $10.5_{-0.5}^{+0.3}$ \\
& $( \pm 0.2)$ & $( \pm 0.04)$ & $( \pm 0.1)$ & \\
\hline GAUSS & & & & \\
$E_{l}[\mathrm{keV}]$ & 6.36 & - & 6.43 & 6.40 \\
& $( \pm 0.02)$ & & $( \pm 0.02)$ & $( \pm 0.02)$ \\
$E W[\mathrm{eV}]$ & $242_{-108}^{+124}$ & - & $275_{-85}^{+95}$ & $190_{-65}^{+75}$ \\
\hline ABSORI & & & & \\
$\xi^{a}$ & - & - & - & $26 \pm 6$ \\
\hline MEKAL & & & & \\
$k T[\mathrm{keV}]$ & - & - & - & 0.18 \\
& & & & \pm 0.03 \\
$\chi_{\text {red }}^{2} / \mathrm{d} . \mathrm{d} . \mathrm{f}$. & $1.08 / 596$ & $1.11 / 230$ & $1.01 / 901$ & $1.06 / 1042$ \\
$f_{2-10 \mathrm{keV}}^{b}$ & 2.8 & 42.7 & 4.3 & 6.3 \\
$L_{2-10 \mathrm{keV}}^{c}$ & 4.8 & 0.17 & 1.2 & 2.3 \\
\hline
\end{tabular}

Model fits were performed to the joint $\mathrm{PN}+\mathrm{MOS} 1+2$ spectra in the $0.4-10 \mathrm{keV}$ range. Errors are at the $90 \%$ level for one interesting parameter; ${ }^{\dagger}$ in units of $10^{20} \mathrm{~cm}^{-2}$; ${ }^{*}$ PL normalization at $1 \mathrm{keV}$ in $10^{-4}$ photons $\mathrm{cm}^{-2} \mathrm{~s}^{-1} \mathrm{keV}^{-1}$; ${ }^{a}$ the ionization parameter which determines the absorber ionization state, and is defined as $\xi=L /\left(n R^{2}\right) ;{ }^{b}$ in units of $10^{-12} \mathrm{erg} \mathrm{cm}^{-2} \mathrm{~s}^{-1} ;^{c}$ in units of $10^{41} \mathrm{erg} \mathrm{s}^{-1}$.

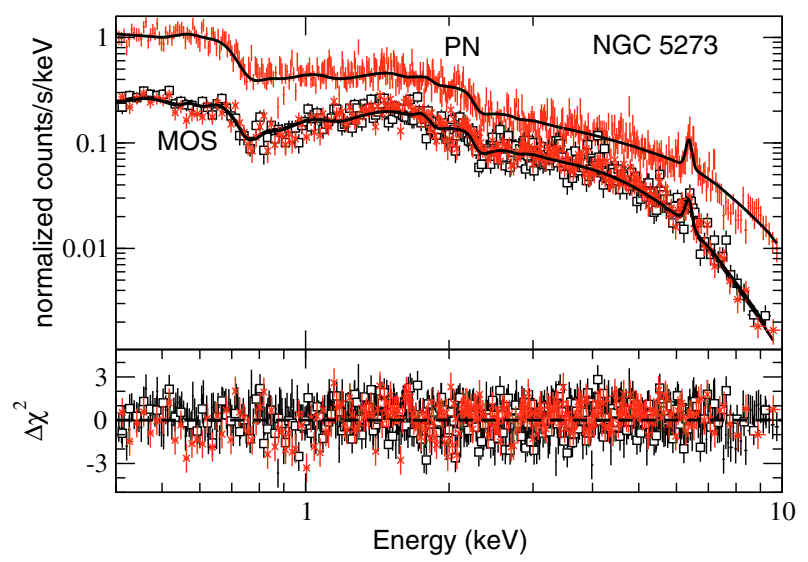

Fig. 4. Simultaneous model fit to the PN, MOS1 (open squares) and MOS2 (asterisks) fit to the NGC $52730.4-10 \mathrm{keV}$ data. The model shown here suggests that the flux from the central engine and warm circumnuclear material is absorbed by an ionized absorber, as well as by cold galactic material in the source. An Fe K line at $6.4 \mathrm{keV}$ can also be seen.

material, (i.e. by a WABS $\times$ ABSORI $\times($ MEKAL + PL + GAUSS $)$ model in XSPEC terminology; the ABSORI power-law photon index was kept proportional to the PL slope).This model yielded a $\chi^{2}$ of 1.05 for 1043 d.o.f. The soft thermal flux in the $0.5-2 \mathrm{keV}$ band accounts for about $10 \%$ of the soft power law flux. Figure 4 shows the abovementioned best model-fit to the EPIC data, together with the best fit residuals.

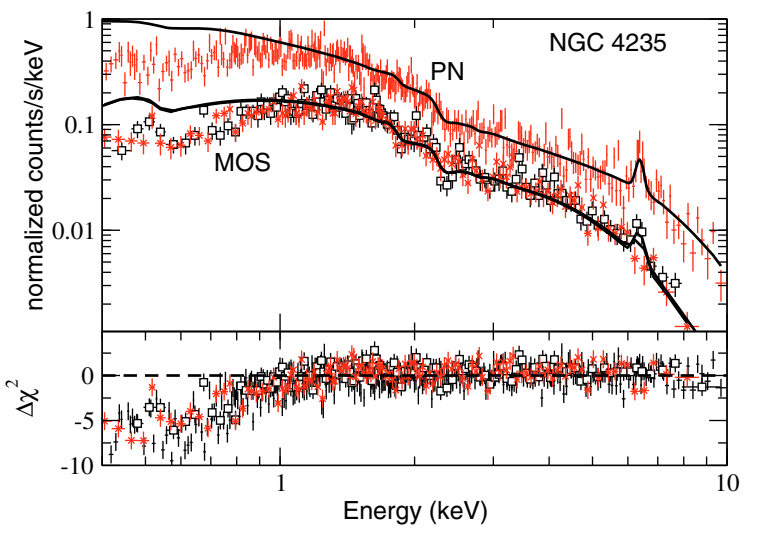

Fig. 5. The 0.4-10 keV EPIC spectra of NGC 4235, plotted together with the $2-10 \mathrm{keV}$ best model fitting "power-law plus Gaussian line" model (upper panel). The model is extrapolated to lower energies assuming Galactic absorption only. The respective model fitting residuals plot is shown in the lower panel of the figure (symbols are as in Fig. 4).

\subsubsection{NGC 4235}

The XMM-Newton observation of this source has not been studied in the past. A simple PL model with $\Gamma \sim 1.6$ plus a $\sim 6.4$ Gaussian line fits the two MOS and the PN spectra well in the $2-10 \mathrm{keV}$ band. Figure 5 shows the spectra together with the $2-10 \mathrm{keV}$ best fitting power law model extrapolated to low energies, assuming Galactic absorption only (upper panel) and the respective best model fitting residuals (lower panel). The flux deficit at energies below $\sim 1 \mathrm{keV}$ clearly indicates the presence of intrinsic absorption in this object.

A PL model plus a Gaussian line plus a cold absorption component (WABS in XSPEC) with $N_{\mathrm{H}}$ left as a free parameter gives acceptable fits to the full band EPIC spectra of the source. The best model fitting results are listed in Table 3 . The inclusion of a Gaussian line improves the simple PL model fit substantially in the case of the PN spectrum only $\left(\Delta \chi^{2}=22.3\right.$ for 2 additional parameters). The iron line is not required in the case of the MOS data, because of their lower statistical quality.

\section{The near-IR to X-ray SEDs}

In order to construct broad band SEDs we use the results from the spectral analysis of the XMM-Newton data, nuclear flux measurements from high-resolution HST images (Sect. 3.1), as well as near-IR measurements from the literature.

For the near-IR data, we use the nuclear flux measurements of Alonso-Herrero et al. (2003), derived from high-resolution HSTNICMOS images at $1.1 \mu \mathrm{m}(F 110 \mathrm{~W}$ filter $)$ and $1.6 \mu \mathrm{m}$ ( $F 160 \mathrm{~W}$ filter). We also use their nuclear flux measurements in the $K\left(\lambda_{\text {central }}=2.12 \mu \mathrm{m}\right)$ and $L$-band $\left(\lambda_{\text {central }}=3.51 \mu \mathrm{m}\right)$, derived from $\sim$ arcsec resolution images taken by the $3.8 \mathrm{~m}$ NASA IRTF telescope on Mauna Kea. The near-IR data are summarized in Table 4. Luminosities at each waveband have been estimated as described in Sect. 2.3.

Finally, we searched the MAST archive at STScI for all available WFPC2 images for the four galaxies, in order to measure their nuclear fluxes in as many as possible optical wavebands. We describe below the HST data selection and reduction methods we have used. 
Table 4. Near-IR and optical-UV fluxes.

\begin{tabular}{lrrrc}
\hline \hline Object & $\lambda(\AA)$ & Flux $^{1}$ & $\log \left(L^{2}\right)$ & Refs. \\
\hline NGC 4235 & $3.5 \mu \mathrm{m}$ & $2.21 \times 10^{-16}$ & 42.13 & 1 \\
& $2.1 \mu \mathrm{m}$ & $<4.49 \times 10^{-16}$ & $<42.21$ & 1 \\
& $1.6 \mu \mathrm{m}$ & $4.33 \times 10^{-16}$ & 42.08 & 1 \\
& $6030 \AA$ & $2.33 \times 10^{-16}$ & 41.62 & 2 \\
NGC 4639 & $5487 \AA$ & $3.32 \times 10^{-17}$ & 39.87 & 2 \\
& $4316 \AA$ & $4.50 \times 10^{-17}$ & 39.90 & 2 \\
& $3004 \AA$ & $2.32 \times 10^{-16}$ & 40.45 & 2 \\
NGC 5033 & $3.5 \mu \mathrm{m}$ & $2.11 \times 10^{-16}$ & 41.31 & 1 \\
& $2.1 \mu \mathrm{m}$ & $<5.45 \times 10^{-16}$ & $<41.50$ & 1 \\
& $1.6 \mu \mathrm{m}$ & $3.78 \times 10^{-16}$ & 41.22 & 1 \\
& $1.1 \mu \mathrm{m}$ & $1.51 \times 10^{-16}$ & 40.66 & 1 \\
& $6030 \AA$ & $1.04 \times 10^{-16}$ & 40.24 & 2 \\
& $5487 \AA$ & $1.08 \times 10^{-15}$ & 41.21 & 2 \\
& $4316 \AA$ & $1.51 \times 10^{-15}$ & 41.26 & 2 \\
NGC 5273 & $3.5 \mu \mathrm{m}$ & $7.35 \times 10^{-17}$ & 40.97 & 1 \\
& $1.6 \mu \mathrm{m}$ & $1.96 \times 10^{-16}$ & 41.06 & 1 \\
& $1.1 \mu \mathrm{m}$ & $1.69 \times 10^{-16}$ & 40.83 & 1 \\
& $7898 \AA$ & $1.44 \times 10^{-16}$ & 40.62 & 2 \\
& $6030 \AA$ & $2.86 \times 10^{-16}$ & 40.80 & 2 \\
& $5487 \AA$ & $3.60 \times 10^{-16}$ & 40.87 & 2 \\
& $4316 \AA$ & $5.43 \times 10^{-16}$ & 40.93 & 2 \\
& $3004 \AA$ & $6.73 \times 10^{-16}$ & 40.87 & 2 \\
\hline & & & &
\end{tabular}

${ }^{1}$ In erg cm ${ }^{-2} \mathrm{~s}^{-1} \AA^{-1} ;^{2}$ in erg s ${ }^{-1}$. References: (1) Alonso-Herrero et al. (2003); (2) this work.

Table 5. Summary of the HST observations.

\begin{tabular}{ccccc}
\hline \hline Object & $\begin{array}{c}\text { Observation } \\
\text { date }\end{array}$ & Filter & $\begin{array}{c}\text { Exp. } \\
(\mathrm{s})\end{array}$ & $\begin{array}{c}\text { Prop. } \\
\text { ID \# }\end{array}$ \\
\hline NGC 4235 & $04 / 29 / 1995$ & $F 606 W(6030 \AA)$ & 500 & 5479 \\
NGC 4639 & $01 / 15 / 1995$ & $F 547 M(5487 \AA)$ & 230 & 5381 \\
& $01 / 15 / 1995$ & $F 439 W(4316 \AA)$ & 700 & 5381 \\
& $01 / 15 / 1995$ & $F 300 W(3004 \AA)$ & 1000 & 5381 \\
NGC 5033 & $05 / 28 / 2001$ & $F 606 W(6030 \AA)$ & 400 & 8597 \\
& $12 / 18 / 1994$ & $F 547 M(5487 \AA)$ & 230 & 5381 \\
& $12 / 18 / 1994$ & $F 439 W(4316 \AA)$ & 700 & 5381 \\
NGC 5273 & $04 / 06 / 1997$ & $F 791 W(7898 \AA)$ & 40 & 6419 \\
& $07 / 04 / 2000$ & $F 606 W(6030 \AA)$ & 400 & 8597 \\
& $01 / 14 / 1995$ & $F 547 M(5487 \AA)$ & 230 & 5381 \\
& $01 / 14 / 1995$ & $F 439 W(4316 \AA)$ & 700 & 5381 \\
& $01 / 13 / 1995$ & $F 300 W(3004 \AA)$ & 1000 & 5381 \\
\hline
\end{tabular}

\subsection{The HST observations}

All four Seyferts were observed in at least one filter with WFPC2 on board HST. A summary of the HST observations we used is given in Table 5. The date of observation, exposure time and HST proposal number are listed in Cols. 2, 4 and 5. Column 3 lists the WFPC2 filters, together with the respective $\lambda_{\text {central }}$ (in parenthesis). For the galaxies with more than one image in each filter, we chose to study the one with the largest exposure that did not cause saturation effects in the central region.

The WFPC2 images had been already processed by the standard STScI reduction pipeline (Baggett et al. 2002). Since we have chosen images with no saturation effects, the only additional processing step required is the removal of cosmic rays. For that reason, we used the filter/cosmic task of the MIDAS

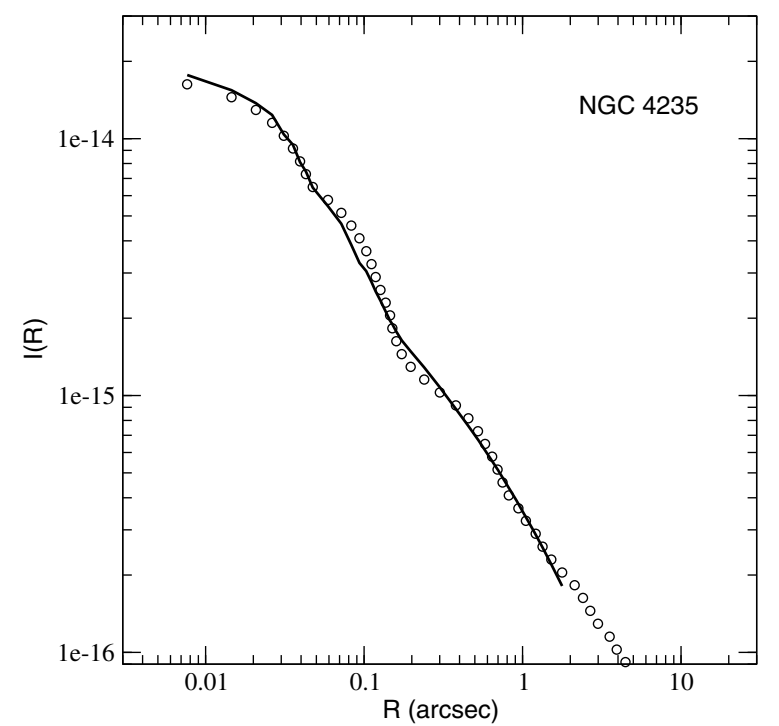

Fig. 6. Radial brightness profile of the central arcsec of NGC 4235 (from the $F 606 \mathrm{~W}$ image). The solid line shows the best fit to the profile (the fit was performed to the innermost 2 " only).

astronomical package. Any residual cosmic ray events as well as bright foreground stars were removed by hand.

In order to measure nuclear fluxes we derived the radial brightness profile of each galaxy. To this end, we performed ellipse fitting to the isophotes of the galaxy images. This choice is motivated by the fact that the isophotes of galaxies, especially elliptical (E) and lenticular (S0) as well as the bulge of spiral galaxies, are not far from ellipses. This technique has been widely used in the past by various authors, mainly as a method of retrieving embedded galaxy structures that are hidden by the large-scale distribution of light of the main body of the galaxy (see Xilouris \& Papadakis 2004, and references therein). Using the fit/ell3 task of MIDAS, which is based on the formulas of Bender \& Möllenhoff (1987), we fitted the isophotes of the galaxy with ellipses and thus derived the average surface brightness profile along the major axis of the galaxy. To convert from counts to physical units, we multiplied the pixel counts by the value of the keyword PHOTFLAM in the image headers and divide by the value of the EXPTIME keyword.

We also created synthetic PSFs using the Tiny Tim software (Krist 1995), which, for WFPC2, produces an accurate representation of the central region of the PSF, thus appropriate for our purpose. We aligned the synthetic PSFs with the position of the nucleus, and constructed the radial profile of the PSFs with the same method as we do for the galaxies.

Since we are not interested in modeling the bulge of the galaxies on large scales, we considered the radial brightness profile only in the central 2 " and we fitted it with a "Sersic's function + PSF" model, i.e. a model of the form $I(R)=$ $I(0) \exp \left\{-\left(R / R_{0}\right)^{1 / n}\right\}+K \times \operatorname{PSF}(R)$, where $I(R)$ is the surface brightness, $I(0), R_{0}$, and $n$ are constants to be determined during the fitting process, $\operatorname{PSF}(R)$ is the PSF radial profile, and $K$ is a constant that determines the unresolved nuclear flux. Figure 6 shows as an example the brightness profile of the innermost $5^{\prime \prime}$ in NGC 4235, together with the best model fitting line.

Our results are listed in Table 4. The nuclear fluxes are corrected for Galactic extinction, adopting the values of Schlegel et al. (1998). In the case of NGC 4235 we also considered intrinsic absorption, given the clear presence of a dust lane in its HST image and the X-ray spectral fitting results which indicate 


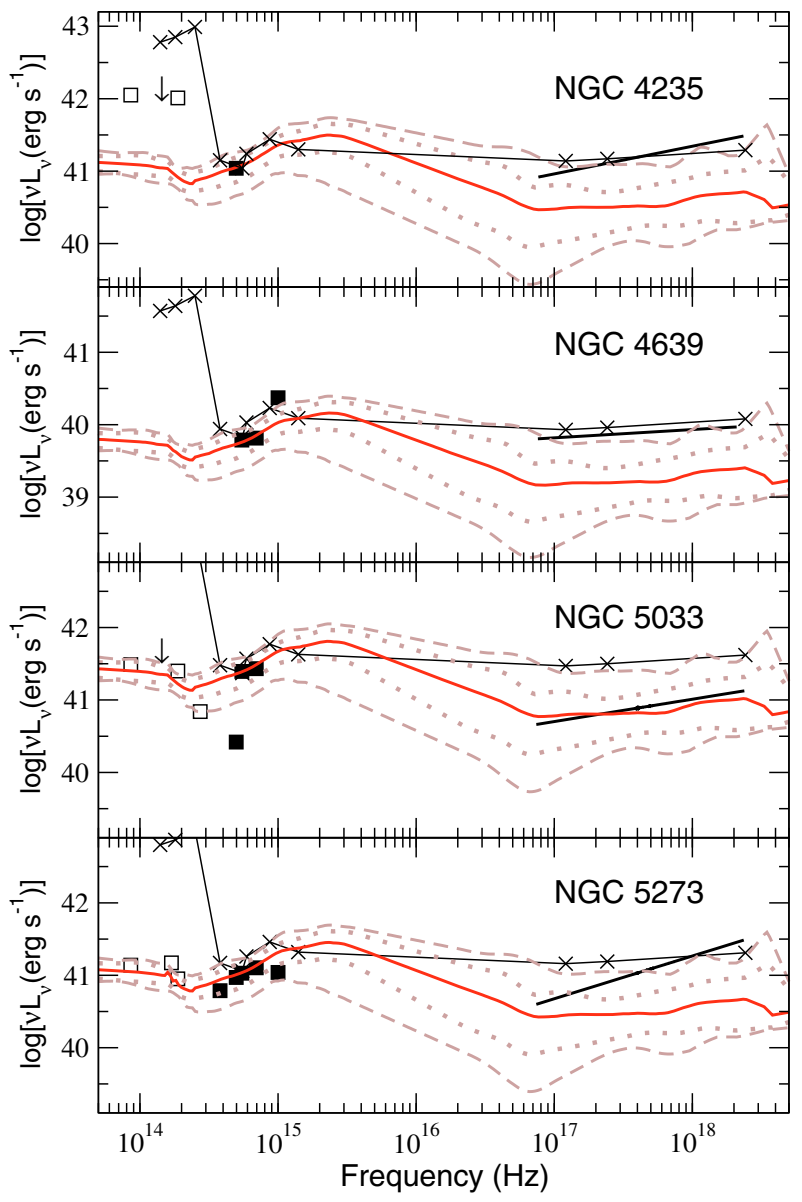

Fig. 7. The near-IR to X-ray SED for the four objects in our sample. Upper limits are $3 \sigma$. The solid, dotted and dashed lines indicate the mean radio-quiet quasar SED from Elvis et al., and the the 68 and 90 percentile contours on each side. Filled squares are our measurements from the HST data, open squares are IR data from the literature. Crosses indicate the nuclear SED of NGC 3998 (see text for details).

absorption of the nuclear source by material within the galaxy. For the reddening correction in the optical band we converted the best fitting $N_{\mathrm{H}}$ value to $A_{V}$ using the relation $A_{V}=N_{\mathrm{H}} / 2.2 \times 10^{21}$ (Ryter 1996), and we found the extinction at $6030 \AA$ using the relations of Cardelli et al. (1989).

Ho \& Peng (2001) have also used the WFPC2/F547M HST images of NGC 4639, NGC 5033, and NGC 5273 to determine their nuclear flux, using an advanced method for galaxy image decomposition. They report a nuclear flux of $3.9 \times 10^{-17}$, $9 \times 10^{-16}$, and $2.7 \times 10^{-16} \mathrm{erg} \mathrm{cm}^{-2} \mathrm{~s}^{-1}$, respectively. These estimates are in agreement with our results.

\subsection{The overall SEDs}

Figure 7 shows the near IR-to-X-ray SEDs of the four Seyferts. The solid line is the average SED of radio-quiet quasars from Elvis et al. (1994); dotted and dashed lines show the 68 and 90 percentile contours on each side of the mean SED, respectively. The average quasar SED is normalized to the flux of NGC 4235 in the $F 606 W$ filter, and to the flux of the other three objects in the $F 439 \mathrm{~W}$ filter. Note that all the measurements plotted in Fig. 7 are non-simultaneous. They were taken several years apart, and variations (by factors of a few, especially in the $\mathrm{X}$-ray band) over these time scales are common in AGN. This fact could introduce uncertainties which should be kept in mind when comparing the non-simultaneous measurements with the mean quasar SED.

The near IR/optical SEDs of NGC 5033 and NGC 5273 show a concave spectrum, which is almost identical, in shape, to the mean quasar SED. This spectral shape is often interpreted as the signature of the presence of the "standard", geometrically thin/optically thick disc emission and of dust heated by the central engine. In NGC 4639 we observe the optical part of the spectrum only, which is rising towards the UV (as expected if we observe thermal emission from the accretion disc).

In the case of NGC 4235 and NGC 5033, the X-ray to optical luminosity ratio is almost identical to what we observe in quasars. In NGC 4639 and NGC 5273, the optical-UV bump appears to be weaker than the X-rays by a factor of a few. Since it is in the X-ray band that AGN show their largest amplitude variations, this difference may not be intrinsic. For example, during its XMM-Newton observation, NGC 4639 appears to be $\sim 3$ and $\sim 10$ times brighter than during its ASCA (Terashima et al. 2002) and CHANDRA observation (Ho et al. 1999).

On the other hand, this difference may be intrinsic, indicating that accretion in these objects operates in a mode that is different to that in powerful quasars. To illustrate this point, the crosses in all panels of Fig. 7 show the nuclear SED of NGC 3998. Data were taken from Table 4 of Ptak et al. (2004). If there were multiple measurements at a certain wavelength, we chose the ones derived from the smallest aperture size. The NGC 3998 SED is rescaled to the optical luminosity of the objects in our sample. Figure 7 shows that the optical to X-ray SEDs of NGC 3998, NGC 4235 and NGC 4639 (at least) are almost identical.

The NGC 3998 SED is consistent with RIAF models. Ptak et al. (2004) found that, although a "pure" thin-disc model does not account for the mid-IR emission in NGC 3998, a truncated thin-disc (at around $\sim 300$ Schwarzschild radii) surrounding an inner RIAF fitted its mid-IR to X-ray spectrum well (when the accretion rate is $\sim 10^{-3}$ of the Eddington limit). Consequently, the qualitative comparison of the (rather sparse) SEDs of the objects in our sample with either the average SED of the radio-quiet quasars or SEDs that are consistent with RIAF models, does not allow us to reach solid conclusions regarding the accretion mode in these objects.

We reach more solid results when we compare the optical and X-ray luminosity in quasars and LLAGNs in a more quantitative way. Figure 8 shows the monochromatic luminosity $L_{\mathrm{V}}$ at $5400 \AA$, plotted as a function of $L_{X}$, for PG quasars (data taken from Elvis et al. 1994). Filled squares show the monochromatic luminosity at $5487 \AA$ plotted as a function of $L_{X}$ in the fours Seyferts of our sample (using the data listed in Tables 3 and 4), while the open squares show data for other nearby Seyferts, using the nuclear flux $5487 \AA$ and X-ray flux measurements of Ho $\&$ Peng (2001) and Cappi et al. (2006). The cross in the same figure indicates the $5000 \AA$ and average $2-10 \mathrm{keV}$ luminosity of NGC 3998, estimated using the flux measurements of Ptak et al. 2004 (as listed in their Table 2) and assuming a distance of 14.1 Mpc.

We find that a line of the form $L_{\mathrm{V}} \propto 1.1( \pm 0.1) L_{\mathrm{X}}$ (solid line in Fig. 8) fits the quasar data well. The dashed line in the same figure shows its extrapolation to lower luminosities. The agreement between the low luminosity Seyferts and the powerful PG quasars is excellent. We do not observe any sign of a "dichotomy" in this plot, over seven orders of magnitude in X-ray luminosity, which might indicate that the low luminosity AGN accrete in the inefficient radiative regime. In fact, the addition of the low luminosity objects seems to strengthen the "optical vs. 


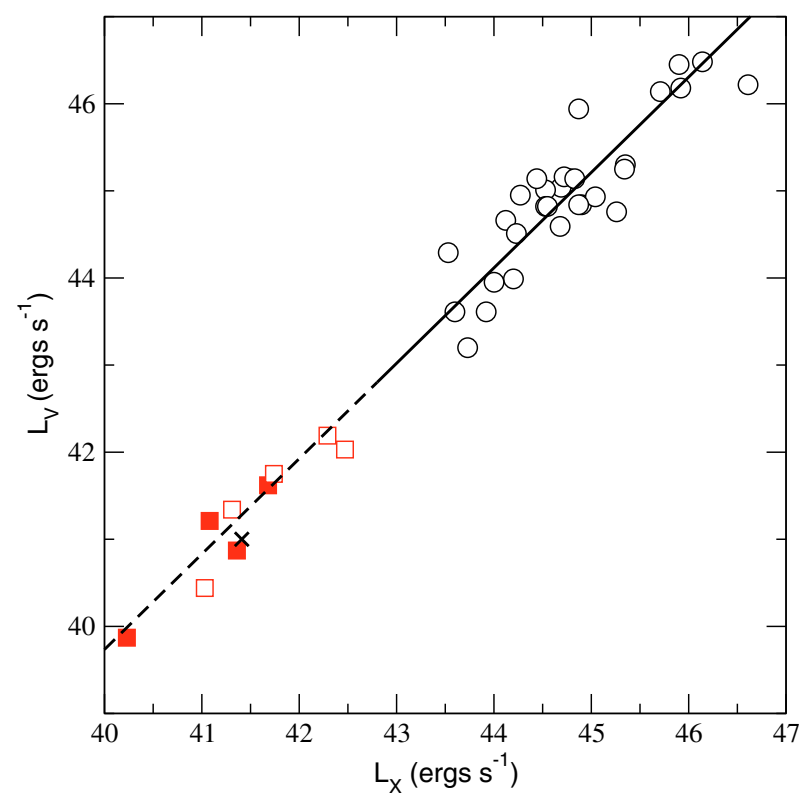

Fig. 8. The $L_{\mathrm{V}}$ versus $L_{\mathrm{X}}$ plot for PG quasars (open circles), nearby Seyferts (open squares), the four Seyferts in our sample (filled squares), and NGC 3998 (cross). The solid line indicates the best linear fit to the quasar sample, and the dashed line is its extrapolation to lower luminosities.

X-ray luminosity" correlation we detect among the PG quasars alone. We conclude that the optical to Xray SEDs of the four objects in our sample (and other nearby, low luminosity Seyferts) is not different from the mean SED of quasars, which are two to five orders of magnitude more luminous.

\section{Discussion and conclusions}

We have used archival XMM-Newton and ASCA data in order to measure the X-ray variability amplitude of four low luminosity Seyfert 1-1.5 galaxies, and compare it with the amplitude of brighter AGN. We have used the same XMM-Newton data to determine accurately their nuclear X-ray luminosity and spectral shape, and HST data to measure the optical/UV flux of their active nucleus. We then combined the XMM-Newton spectra, the HST measurements, and near-IR data from the literature to construct their broad band, near-IR to X-ray SED and compare it with the mean SED of radio-quiet quasars. Our main aim is to investigate whether accretion in these objects operates in a mode which is different from the accretion mode in their more luminous counterparts.

Such comparisons are frequently used to investigate the nature of the nuclear source in low luminosity AGNs. Most of the similar work in the past has focused mainly on the comparison between the properties of galaxies with low-ionization nuclear emission-line regions (LINERs; Heckman 1980) and luminous AGN. However, the nature of LINERS and their relation to AGN has been debated for several years. For this reason, we decided to study objects with a firm optical classification as a Seyfert 11.5 galaxy. In this way, we are certain that they host an accretion driven, AGN-like, UV to X-ray nuclear source. In addition, the clear presence of broad lines in their optical spectra implies that we can view their central source directly, and hence determine their nuclear SED reliably.

Furthermore, using the X-ray luminosities listed in Table 3, and the BH mass estimates listed in Table 2, we find that the
$\left(L_{\mathrm{X}} / L_{\text {Edd }}\right)$ ratio of NGC 5273 is $6 \times 10^{-4}$. The value of the same ratio in the case of NGC 4235, NGC 4639 and NGC 5033 is even smaller: $7.7,1.8$ and $3.3 \times 10^{-5}$, respectively. This is much smaller than the same ratio of powerful quasars, and even 20 to 80 times smaller than the ratio of other Seyfert 1 galaxies in the Palomar survey (for example NGC 4051, NGC 4151 and NGC 5548). It is also below the limit of $10^{-3}$ where Merloni et al. (2003) suggest that the accretion mode may change from a thin, optically thick disc to that of a RIAF. We also converted the measured F547 fluxes of NGC 4639, NGC 5033 and NGC 5273, and the measured F606W flux of NGC 4235, to flux at $7000 \AA$, assuming an optical spectral index of 1 , and we estimated the monochromatic luminosity at this wavelength. Chiaberge et al. (2005) suggest that if $L_{\mathrm{O}} / L_{\mathrm{Edd}} \leq 10^{-4}$, then a RIAF may operate in an AGN. In the case of NGC 4235, NGC 4639 and NGC 5033, this ratio is $6-60 \times 10^{-6}$, while in the case of NGC $5273, L_{\mathrm{O}} / L_{\mathrm{Edd}} \sim 1.5 \times 10^{-4}$.

In summary, the four objects of our study i) should host a relatively unobscured AGN-like nucleus; ii) are of low luminosity; and iii) accrete at a low rate as well. Hence they are good candidates to detect radiation from RIAF-like processes.

We detect significant X-ray variations in the XMM-Newton light curves of NGC 5033 and NGC 5273, and in the ASCA light curve of NGC 4235 . The variability amplitude is $5-15 \%$ of the light curve's mean, and is of the "right" order, given their BH mass. In agreement with Ptak et al. (1998), we find that these objects do not follow the "variability amplitude - luminosity" relation of other more luminous AGN. However, this result does not necessarily imply a larger characteristic size for the X-ray producing region than is the case in "normal" AGN. On the contrary, if the size is determined by $\mathrm{BH}$ mass, as expected, then our results suggest that, when scaled to $\mathrm{BH}$ mass, the size of their X-ray sources is similar to the size of the nuclei in more luminous AGN. Their "misplacement" in the "amplitude - luminosity" plane is not due to their variability amplitude being too low but due to their luminosity being low, most probably because of their low accretion rate. Their X-ray spectral shape is also consistent with the shape of more luminous AGN. Even the best fitting spectral slope in the case of NGC 5273 is not exceptionally unusual; NGC 4151, a well studied, luminous Seyfert galaxy, also shows a very flat X-ray spectrum of $\Gamma \sim 1.5$ (see e.g. de Rosa et al. 2007). We therefore conclude that, from the X-ray point of view, the central engine in these low luminosity Seyferts operates in the same way as in the more luminous AGN.

The near-IR to X-ray SEDs of the objects in our sample are rather sparse, and their qualitative comparison with either the average SED of radio-quiet quasars or the SED of other lowluminosity objects which appear to host a RIAF is not conclusive. If we take into account the fact that we used measurements taken several years apart, and the possibility of large amplitude variations, at least in the X-ray band, then it is reasonable to conclude that the SEDs of the AGN in our sample are similar to the average SED of quasars. On the other hand, the SED of at least NGC 4235 and NGC 4639 are almost identical to the SED of NGC 3998, which is consistent with a model of a truncated disc and a RIAF at smaller radii (see also the case of NGC 4579 and M 81 whose SEDs are consistent with a similar model; Quataert et al. 1999).

The situation becomes clearer when we compare the optical luminosity, $L_{\mathrm{V}}$, with the X-ray luminosity, $L_{\mathrm{X}}$, in the 4 Seyferts of our sample (and a few other LLAGNs) and in quasars (i.e. AGN which are $10^{2}$ to $10^{5}$ times more luminous). We find that the quasar optical luminosity increases almost proportionally with $L_{\mathrm{X}}$. The extrapolation of the quasar " $L_{\mathrm{V}}$ vs. $L_{\mathrm{X}}$ " best fit line 
to lower luminosities agrees very well with the LLAGN data. It is then reasonable to assume that the observed "continuity" in the $\left(L_{\mathrm{V}}, L_{\mathrm{X}}\right)$ distribution of quasars and LLAGNs also suggests that the optical/X-ray SEDs in AGN are similar, hence there is no accretion mode "phase transition" over almost seven orders of magnitude.

Our results are in agreement with the results of Panessa et al. (2006) who study the X-ray and optical emission line correlation in a sample of AGN with a wide range of Eddington ratios, and conclude that low-luminosity Seyferts are simply scaleddown versions of luminous quasars. Similar results were obtained by Maoz (2007) who studied the SEDs of 13 low luminosity LINERs, and found that radiatively efficient discs may operate even in these objects, which are characterized by very low accretion rates.

Although we have tried to use the best available data to determine the SED for the four Seyferts, the fact that the measurements we use in the various bands are separated by years introduces some uncertainty. Significant improvement can be achieved if we manage to determine the average nuclear flux of each object in various bands. This can be achieved only if we observe individual objects many times over a time scale of a few years at least, which is not an easy task. However, the "variability amplitude test" results are not affected by these limitations. Even if we consider only them, the main result of our work is that accretion operates in the "normal" way even in objects where the ratio $L_{\mathrm{X}} / L_{\text {Edd }}$ is as low as a few $\times 10^{-5}$.

Perhaps it is not just the accretion rate that determines the accretion mode in AGN. Black hole spin and/or mass may also play a role in how accretion operates in active galaxies. We plan to investigate this issue by studying the variability properties of a large sample of low luminosity AGNs, with a wide range of $\mathrm{BH}$ masses. A dichotomy in the "variability amplitude - BH mass" plane could provide us with important clues to what the main parameters are that dictate the "switch" in the AGN accretion mode.

Acknowledgements. This work is based on observations with XMM Newton, an ESA science mission with instruments and contributions directly funded by ESA Member States and the USA (NASA). It has made use of the NASA/IPAC Extragalactic Data Base (NED) which is operated by the Jet Propulsion Laboratory, California Institute of Technology, under Contract with the National Aeronautics and Space Administration, and of the Tartarus (Version 3.1) database, created by Paul O'Neill and Kirpal Nandra at Imperial College London, and Jane Turner at NASA/GSFC. Tartarus is supported by funding from PPARC, and NASA grants NAG5-7385 and NAG5-7067. We gratefully acknowledge travel support through the bilateral Greek-German IKYDA2004 personnel exchange program.

\section{References}

Alonso-Herrero, A., Quillen, A. C., Rieke, G. H., Ivanov., V. D., \& Efstathiou, A. 2003, AJ, 126, 81

Baggett, S., et al. 2002, in HST WFPC2 Data Handbook, v. 4.0, ed. B. Mobasher, Baltimore, STScI

Bender, R. F., \& Möllenhoff, C. 1987, A\&A, 177, 71

Cappi, M., Panessa, F., Bassani, L., et al. 2006, A\&A, 446, 459

Cardelli, J. A., Clayton, G. C., \& Mathis, J. S. 1989, ApJ, 345, 245

Chiaberge, M., Capetti, A., \& Macchetto, F. D. 2005, ApJ, 625, 716

Chiaberge, M., Gilli, R., Macchetto, F. D., \& Sparks, W. B. 2006, ApJ, 651, 728

de Rosa, A., Piro, L., Perola, G. C., et al. 2007, A\&A, 463, 903

Dickey, J. M., \& Lockman, F. J. 1990, ARA\&A, 28, 215

Dong, X. Y., \& de Robertis, M. M. 2006, AJ, 131, 1236

Ehle, M., Breitfellner, M., Diaz Trigo, M., et al. 2007, XMM-Newton Users Handbook, http://xmm.esac.esa.int/external/xmm_user_ support/documentation/uhb_2.5/

Elvis, M., Wilkes, B. J., McDowell, J. C., et al. 1994, ApJS, 95, 1

Heckman, T. M. 1980, A\&A, 87, 152

Ho, L. C. 1999, ApJ, 516, 672

Ho, L. C., \& Peng, C. Y. 2001, ApJ, 555, 650

Ho, L. C., Filippenko, A. V., \& Sargent, W. L. 1995, ApJS, 98, 477

Ho, L. C., Filippenko, A. V., \& Sargent, W. L. W. 1997, ApJS, 112, 315

Ho, L. C., Ptak, A., Terashima, Y., Kunieda, H., et al. 1999, ApJ, 525, 168

Elvis, M., Wilkes, B. J., \& Lockman, F. J. 1989, ApJS, 95, 413

Krist, J. 1995, in Astronomical Data Analysis Software and Systems IV, ASP, Conf. Ser., 77, 349

Maoz, D. 2007, MNRAS, 377, 1696

Merloni, A., Heinz, S., \& di Matteo, T. 2003, MNRAS, 345, 1057

Nandra, K., George, I. M., Mushotzky, R. F., Turner, T. J., \& Yaqoob, T. 1997, ApJ, 476, 70

O’Neill, P. M., Nandra, K., Papadakis, I. E., \& Turner, T. J. 2005, MNRAS, 358, 1405

Panessa, F., Bassani, L., Cappi, M., et al. 2006, A\&A, 455, 173

Papadakis, I. E. 2004, MNRAS, 348, 207

Ptak, A., Yaqoob, T., Mushotzky, R., Serlemitsos, P., \& Griffiths, R. 1998, ApJ, 501, 37

Ptak, A., Terashima, Y., Ho, L. C., \& Quataert, E., 2004, ApJ, 606, 173

Quataert, E., Di Matteo, T., \& Narayan, R. 1999, ApJ, 525, L89

Ryter, C. E. 1996, Ap\&SS, 236, 285

Schlegel, D. J., Finkbeiner, D. P., \& Davis, M. 1998, ApJ, 500, 525

Shakura, N. I., \& Sunyaev, R. A. 1973, A\&A, 24, 337

Terashima, Y., Iyomoto, N., Ho, L., \& Ptak, A. F. 2002, ApJS, 139, 1

Vaughan, S., Edelson, R., Warwick, R. S., \& Uttley, P. 2003, MNRAS, 345, 1271

Wu, X.-B., \& Han, J. L. 2001, A\&A, 380, 31

Xilouris, E. M., \& Papadakis, I. 2002, A\&A, 387, 441

Yuan, F. 2007 in: The Central Engine of AGN, ASPS Conf. Ser., 373, 95 